\title{
The Suitability of Compatibility Test Results with Gel Method between Diagnostic Grifols Gel Coombs and Diamed-Identification
}

\author{
Tigor Pandapotan Sianturi, Betty Agustina Tambunan
}

Department of Clinical Pathology, Faculty of Medicine, Airlangga University/Dr. Soetomo Hospital, Surabaya, Indonesia. E-mail: sianturiyudika@gmail.com

\section{ABSTRACT}

A compatibility test as part of a pre-transfusion test is mandatory to ensure blood compatibility between patients and donors. Diamed-ID as the first gel-based product is commonly used as a reference for the compatibility test. The presence of new products such as DG Gel Coombs encourages research to compare them with reference methods. This study aimed to analyze the suitability of DG Gel Coombs to Diamed-ID in the compatibility test with the same sample. This cross-sectional analytic observational study was conducted during November 2017-February 2018 at the Blood Transfusion Unit Dr. Soetomo Hospital, Surabaya using blood samples $(n=40)$, which met the inclusion and exclusion criteria. Simultaneous testing of the two products was according to the manufacturing requirements of each product (using different LISS reagents for red blood cell suspensions and centrifugation arrangements). The suitability of results was tested with Cohen's kappa and significant differences with McNemar. There was a minimum suitability of DG Gel Coombs to Diamed-ID for major compatibility tests, $\mathrm{k} 0.307$ (95\% CI: -0.029-0.643), significance 0.007 ( $\mathrm{p}<0.05)$ and moderate for minor, $\mathrm{K} 0,678(95 \% \mathrm{CI}$ : $0.454-0.903$ ), significance $<0.0001$ ( $p<0.05$ ). McNemar's significance was 0.016 ( $<0.05)$ for major compatibility test and 0.031 ( $p<0.05)$ for minor. Referring to Diamed-ID's results, false negatives were found on DG Gel Coombs for major compatibility tests $(n=7)$ and minor $(n=6)$. The suitability of results from DG Gel Coombs and Diamed-ID is not strong for compatibility testing.

Keywords: Compatibility test, gel method, diagnostic Grifols Gel Coombs, Diamed-Identification

\section{INTRODUCTION}

In the current era of modern medicine, blood transfusion is an important component in the clinical management of patients, especially as a life-saving management in various life-threatening health conditions. ${ }^{1}$ Blood transfusions have become a routine procedure in a wide variety of cases, including blood loss replacement during major surgery, childbirth, major accidents, anemia unresponsive to treatment (uncompensated), and it also helps the treatment of congenital blood disorders such as Thalassemia or Sickle cell anemia. ${ }^{1-3}$

Dr. Soetomo Hospital, Surabaya as one of the referral center hospitals in Indonesia carries out daily blood transfusion. ${ }^{4}$ The Hospital Blood Transfusion Unit (UTDRS) conducts compatibility tests on demand for transfused blood and its components as part of the pre-transfusion test to ensure the compatibility between donor blood and patient blood. $^{4-7}$

Compatibility testing procedures must be performed before a blood transfusion to prevent hemolysis, agglutination, and even death due to severe hemolytic anemia in patients. ${ }^{1,5,8-10}$ The compatibility test must be carried out although the test itself is unable to prevent the immunization process in the patient body and does not fully guarantee the normal survival of donor's red blood cells in the patient's body and unable to detect all unexpected antibodies in the patient's serum. ${ }^{11}$

Compatibility test at UTDRS Dr. Soetomo Hospital, Surabaya uses the gel method replacing the conventional tube method. ${ }^{4}$ The gel method was first introduced by Lapierre in 1998 with more advantages over the tube method, such as easy, simple method without washing steps, shorter inspection time, less sample volume, and better safety for workers because sample exposure can be reduced despite costs. This test is relatively more expensive. ${ }^{12-15}$

Diamed-ID (Diamed GMBH, Cressier, Switzerland) is the first product to use the gel method and is widely used as a reference by several referral laboratories and blood banks worldwide, such as PMI (Indonesian Red Cross). ${ }^{12,16,17}$ DG Gel 
Coombs, a new product with the exactly same method (Diagnostic Grifols S.A., Barcelona, Spain), is present as an alternative in daily use of compatibility testing. ${ }^{13}$ Several studies are present to examine the quality of DG Gel Coombs products against reference products, such as Diamed-ID. ${ }^{13,16,17}$ The study by Cid et al. on the accuracy of the pretransfusion test diagnosis between DG Gel Coombs and Diamed-ID concluded that both products had a high estimation of diagnostic accuracy, and DG Gel Coombs provides more advantage with 8 microtubes on each panel, which can increase the number of tests in each cycle. ${ }^{13}$ Taylor et al. study of the suitability of the pre-transfusion test results between DG Gel Coombs and Diamed-ID also showed good compatibility between the two products. ${ }^{16}$ In contrast to the two studies Hustinx et al., found mismatched pre-transfusion test results between DG Gel Coombs and Diamed-ID in a larger number of samples. ${ }^{17}$ Each laboratory can have its policy on the use of certain products by considering the sensitivity, specificity, cost-effectiveness, time, sample volume, or other matters as needed. ${ }^{13}$

UTDRS Dr. Soetomo Hospital, Surabaya is currently using DG Gel Coombs for routine compatibility testing. Research on the suitability of the pre-transfusion test results, a compatibility test with the gel method between DG Gel Coombs and Diamed-ID as a reference product remained limited and showed varying results, including in Dr. Soetomo Hospital. This research is important to determine whether the DG Gel Coombs product can be used as a choice in routine compatibility testing. Based on the description above, the authors performed aimed to perform a compatibility test between DG Gel Coombs and Diamed-ID by comparing the results of both methods.

This study aimed to analyze the suitability of the Diagnostic Grifols (DG) Gel Coombs product with Diamed-Identification (ID) in the compatibility test with the same sample.

\section{METHODS}

This research was an analytical observational study with a cross-sectional design and, was performed in the period of November 2017-February 2018 at UTDRS Dr. Soetomo Hospital, Surabaya.

The target population was inpatients of Dr. Soetomo Hospital, Surabaya who needed a blood transfusion. Accessible population was all inpatients who requested transfusion blood and its components at the UTDRS Dr. Soetomo Hospital, Surabaya. The samples in this study were blood samples from patients and donors who were asked for compatibility tests at the UTDRS Dr. Soetomo Hospital, Surabaya. There were 40 samples in this study it had met the requirement of sample size in this study ${ }^{18}$ The inclusion criteria were blood samples from patients and donors who requested for the compatibility testing for transfusion purposes at the UTDRS Dr. Soetomo Hospital, Surabaya, patients' blood collected in EDTA tubes and donors' blood in bags containing CPDA- 1 anticoagulant and must be freshly collected or collected in less than $3 \times 24$ hour. The exclusion criteria were hemolyzed samples or samples contaminated with intravenous fluids during blood collection. ${ }^{5-7,9}$

The patient's blood sample was collected in the EDTA tube and the donor sample was collected in a blood bag containing the anticoagulant CPDA- $1^{5-7}$ Patients' and donors' blood were centrifuged at 3000 rpm $(1500 \mathrm{~g})$ for ten minutes to separate red blood cells and serum. The red blood cell suspension was then made with Low Ionic Strength Solution/LISS reagent. DG Gel Sol is a LISS reagent on DG Gel Coombs and ID-Diluent 2 on Diamed-ID. A one percent red blood cell suspension (a mixture of $10 \mu \mathrm{L}$ patient and donor red blood cells in $1 \mathrm{~mL}$ DG Gel Sol) was used for the Coombs DG Gel procedure and $0.8 \%$ suspension (a mixture of $10 \mu \mathrm{L}$ patient and donor red blood cells in $1 \mathrm{~mL}$ ID-Diluent 2) for Diamed-ID. ${ }^{19-21}$

A major compatibility test was carried out by inserting $50 \mu \mathrm{L}$ of donors' cell suspension in the first tube and adding $25 \mu \mathrm{L}$ of patients' serum in the same tube. A minor compatibility test was carried out by inserting $50 \mu \mathrm{L}$ of the patient's cell suspension in the second tube and adding $25 \mu \mathrm{L}$ of donor serum in the same tube. The gel card was incubated and centrifuged in the incubator and centrifuge kit (DG Therm, DG Spin for DG Gel Coombs and ID-Incubator, ID-Centrifuge for Diamed-ID) according to the manufacturing requirements of each product. Incubation was carried out for 15 minutes at $37^{\circ} \mathrm{C}$ and centrifugation was set at 990 rpm (128.1 g) for 9 minutes for DG Gel Coombs and $1030 \mathrm{rpm}$ (85 g) for 10 minutes for Diamed-ID. ${ }^{20,21}$

The results of the major and minor compatibility tests performed on these two products were qualitatively assessed in this study based on the presence of red blood cell agglutination along the gel column. A formation of a clear red blood cell deposit at the bottom of the microtube and the gel on top of the clear red blood cell deposit with no agglutination was reported negative. Contrastingly, if agglutination of red blood cell formed along the gel column was reported positive as shown in Figure 1. ${ }^{20,21}$ 

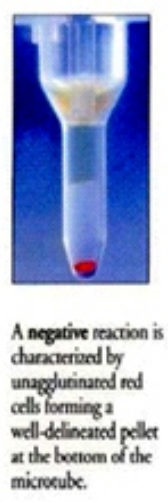

at the bection of the miknoulic.

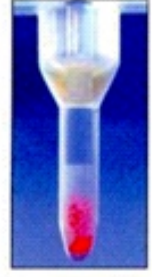

A 1+ reacion is characterined by red cell agducinatos predominuacly obsented in the lower half of the gel columa. Unaptutinated celly form a pellet in the botton of the

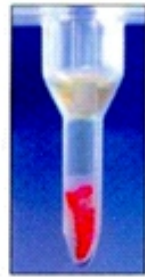

A 2. encrion is chancterined by red cell aglutinate dispened throughour the lenght of the gel column. Fe aplutinato may be of the microeule.

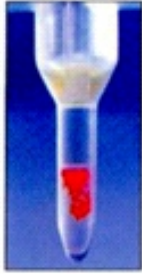

A 3, nexcion is

chancterined by the mipxity of rod cell apglutinutes trapped in the upper half of
gd column.

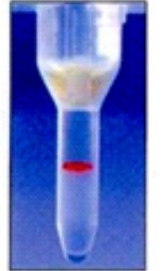

A 4t reaction is charactcriad by a colid band of red cell ayducinates on top of the gid A f fow aplutinates may filcer neat the proberinan band.

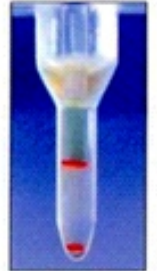

A mixed cell reacion is characterized by a band of red cell ayjuinute on oop of the ged. acoompaniced by a peller of unapgutinated cells us the boetom of the microede.

Figure 1. Interpretation of compatibility testing results with gel method. ${ }^{20,21}$

Positive and negative test results with DG Gel Coombs were compared with test results with Diamed-ID for the same sample. The compatibility between DG Gel Coombs and Diamed-ID was calculated with Cohen's kappa coefficient. Kappa coefficient value of 0.21-0.39 was categorized as minimum suitability, $0.40-0.59$ was categorized as weak suitability, $0.60-0.79$ was categorized as moderate suitability, and $0.80-0.90$ was categorized as strong suitability. The difference in the proportion of positive values of compatibility testing results between DG Gel Coombs and Diamed-ID was determined using the Mc Nemar test with $p<0.05{ }^{18,22}$

This study was approved by the Health Research Ethics Committee of Dr. Soetomo Hospital, Surabaya with ethical number 25/Panke.KKE/I/2018.

\section{RESULTS AND DISCUSSIONS}

Results of major and minor compatibility testing with DG Gel Coombs can be seen in Table 1.

Table 1. Results of compatibility testing with DG Gel Coombs

\begin{tabular}{|c|c|c|}
\hline \multirow[t]{2}{*}{ Result } & \multicolumn{2}{|c|}{$\begin{array}{c}\text { Compatibility Testing with DG Gel } \\
\text { Coombs }\end{array}$} \\
\hline & Major & Minor \\
\hline Positive & 2 (5\%) & $11(27.5 \%)$ \\
\hline Negative & 38 (95\%) & 29 (72.5\%) \\
\hline Total & 40 (100\%) & 40 (100\%) \\
\hline
\end{tabular}

The same sample was tested with Diamed-ID and the results of major and minor compatibility testing can be seen in Table 2 .

The compatibility analysis between the results of the major compatibility testing with DG Gel Coombs and Diamed-ID showed positive results in two samples $(22.2 \%)$ and negative results in 31 samples (100\%) with both DG Gel Coombs and Diamed-ID as shown in Table 3.
Table 2. Results of compatibility testing with Diamed-ID

\begin{tabular}{llc}
\hline \multirow{2}{*}{ Result } & \multicolumn{2}{c}{$\begin{array}{c}\text { Compatibility Testing with } \\
\text { Diamed-ID }\end{array}$} \\
\cline { 2 - 3 } & \multicolumn{1}{c}{ Major } & Minor \\
\hline Positive & $9(22.5 \%)$ & $17(42.5 \%)$ \\
Negative & $31(77.5 \%)$ & $23(57.5 \%)$ \\
Total & $40(100 \%)$ & $40(100 \%)$ \\
\hline
\end{tabular}

Table 3. Compatibility of major compatibility testing results

\begin{tabular}{llll}
\hline Mayor DG Gel & \multicolumn{2}{c}{ Mayor Diamed - ID } & \multirow{2}{*}{ Total } \\
\cline { 2 - 3 } \multicolumn{1}{c}{ Coombs } & Positive & Negative & \\
\hline Positive & $2(22.2 \%)$ & $0(0 \%)$ & $2(5 \%)$ \\
Negative & $7(77.8 \%)$ & $31(100 \%)$ & $38(95 \%)$ \\
Total & $9(100 \%)$ & $31(100 \%)$ & $40(100 \%)$ \\
\hline
\end{tabular}

The McNemar test to determine the difference in the proportion of positive values of the DG Gel Coombs major compatibility testing results with Diamed-ID showed a significance value of 0.016 $(p<0.05)$, suggesting that the significant difference in the proportion of positive values between the results of the DG Gel Coombs major compatibility testing and Diamed-ID was not by chance. The Cohen's kappa test to determine the strength of the conformity of the major compatibility test results between DG Gel Coombs and Diamed-ID showed a Kappa coefficient value of 0.307 (95\% CI: -0.029-0.643), which was categorized as minimum conformity with a significance value of 0.007 $(p<0.05)$. The significance value below $\alpha$ indicates minimal compatibility between DG Gel Coombs and Diamed-ID. This minimal conformity indicates that only a few results from the DG Gel Coombs major compatibility test match the reference product (Diamed-ID). ${ }^{18,22}$ 
The compatibility analysis between the DG Gel Coombs and Diamed-ID minor showed positive results in 11 samples (64.7\%) and negative results in 23 samples (100\%) with both DG Gel Coombs and Diamed-ID as shown in Table 4.

Table 4. Compatibility of minor compatibility testing results

\begin{tabular}{llll}
\hline Minor DG Gel & \multicolumn{2}{c}{ Minor Diamed - ID } & \multirow{2}{*}{ Coomb } \\
\cline { 2 - 3 } \multicolumn{1}{c}{ Coombs } & Positive & Negative & \\
\hline Positive & $11(64.7 \%)$ & $0(0 \%)$ & $11(27.5 \%)$ \\
Negative & $6(35.3 \%)$ & $23(100 \%)$ & $29(72.5 \%)$ \\
Total & $17(100 \%)$ & $23(100 \%)$ & $40(100 \%)$ \\
\hline
\end{tabular}

The McNemar test to determine the difference in the proportion of positive values of the DG Gel Coombs minor compatibility testing results with Diamed-ID showed a significance value of 0.031 $(p<0.05)$, indicating that the significant difference in the proportion of positive values between the results of the DG Gel Coombs minor compatibility testing with Diamed-ID was not by chance. The Cohen's kappa test to determine the conformity strength of the minor compatibility test results between DG Gel Coombs and Diamed-ID showed a Kappa coefficient value of 0.678 (95\% CI: $0.454-0.903)$, which was categorized moderate conformity with a significance value of $<0.0001(p<0.05)$. The significance value below $\alpha$ indicates that there is a moderate conformity for the results of the minor compatibility testing between DG Gel Coombs and Diamed-ID. 18,22

Diamed-ID as the first gel-based product for pre-transfusion testing has been recognized for its superiority over conventional tube methods and is currently a reference product in many laboratories. ${ }^{12-15}$ Swarup et al. reported the superiority of the gel method (Diamed-ID) over the conventional tube method for compatibility testing and Bromilow et al. and Knight de Siva, reported for the antibody screening. The study concluded that the gel method was a better substitute for the conventional tube method with several advantages as previously mentioned. . $^{9,12-15}$

DG Gel Coombs as a new product with the gel method is present as a choice in routine pre-transfusion testing including compatibility testing. There have been several studies aimed to analyze the compatibility of the pre-transfusion test results between DG Gel Coombs and Diamed-ID as a reference method. ${ }^{13,16,17}$

Cid et al. reported strong compatibility of the pre-transfusion test results (antibody screening) between DG Gel and Diamed-ID in 3019 samples from a total of 3024 samples. $^{13}$ Taylor et al. also reported strong compatibility of pre-transfusion test results ( $A B O / D$ grouping, antibody screening, $R h$ phenotyping, $K$ antigen determination, antibody identification, $D A T$, and $A B O / D$ tests in neonates) between DG Gel and Diamed-ID in 4182 out of total 4281 samples. $^{16}$ The compatibility of the DG Gel Coombs results with the reference product (Diamed-ID) from those studies concluded that equipment and reagents of DG Gel Coombs were reliable to be used in routine immunohematological laboratory tests, especially pre-transfusion tests. ${ }^{13,16}$ In contrast to the studies of Cid et al., Taylor et al., and Hustinx et al. found a discrepancy in the results of pre-transfusion tests (antibody screening and antibody identification) between DG Gel Coombs and Diamed-ID in 10 samples from a total of 200 samples. ${ }^{17}$

This study also aimed to analyze the compatibility of the pre-transfusion test results between DG Gel Coombs and Diamed-ID. In contrast to the results of previous studies, which showed strong compatibility between DG Gel Coombs and Diamed-ID, this study showed weak or minimum compatibility between DG Gel Coombs and Diamed-ID for major compatibility tests and moderate compatibility for minor compatibility tests. Referring to the Diamed-ID results as a reference method, there were also false-negative results on DG Gel Coombs in seven samples for major compatibility tests and six samples for minor compatibility tests, respectively. Several factors such as constant affinity, $\mathrm{pH}$, ionic strength, temperature, incubation time, and the ratio of serum to red blood cells can interfere with the strength of the antigen-antibody binding in the product of the gel method, resulting in false positive and negative results for reference products. ${ }^{23}$ This false-negative result contributes to the compatibility of the results between the two products. ${ }^{13}$

Taylor et al., stated some manufacturing requirements for each product such as the use of LISS reagents in the manufacture of red blood cell suspensions and different centrifugation settings also influence the results of pre-transfusion tests. ${ }^{16} \mathrm{~A}$ study by Gray et al. showed that the high conductivity of the LISS reagent used in the manufacture of red blood cell suspensions can be the cause of false-negative results in some cases. Increasing the conductivity of the LISS reagent will increase the ionic strength, which in turn causes suboptimal antibody binding during incubation for a short time. ${ }^{23} \mathrm{Cid}$ et al. reported that the conductivity of DG Gel Sol is 5.09 $\mathrm{mScm}-1 .{ }^{13}$ Philips and Bebbington, reported that this 
value was not within the acceptable range of $3.7 \pm 0.3$ $\mathrm{mScm}-1$. Contrastingly, the ID-Diluent 2 conductivity value was known to be in an acceptable range. ${ }^{23}$

Different centrifugation settings to the manufacturing requirements for each product also affect the compatibility of the results. ${ }^{16}$ The use of DG Spin as a centrifuge for DG Gel Coombs, which was set at $990 \mathrm{rpm}(128.1 \mathrm{~g})$ for 9 minutes gives the possibility of a higher sedimentation rate compared to the use of ID-Centrifuge for Diamed-ID, which was set at $1030 \mathrm{rpm}(85 \mathrm{~g})$ for 10 minutes. ${ }^{16,20,21,23,24}$ The increase in Relative Centrifugal Force (RCF) is known to increase the sedimentation rate, which causes the accumulation of sediment in the bottom of the gel. This increases the likelihood of a false negative result on DG Gel Coombs. ${ }^{16,24}$

The limitation of this study was no validation of positive results on both products by screening and antibody identification. Therefore, further research was needed to include both stages of the test in analyzing the compatibility of the results of both products.

\section{CONCLUSIONS AND SUGGESTIONS}

There was minimum compatibility of the major compatibility testing results with the gel method between DG Gel Coombs and Diamed-ID and moderate compatibility for minor compatibility tests. Researchers suggested further research by adding screening tests and antibody identification to analyze the compatibility of the results of both products.

\section{REFERENCES}

1. Blanchet $\mathrm{O}$, Bonte L, Bouhabib H, Chaillet P. Blood transfusion. A manual for doctors, nurses, and laboratory technicians. Paris, Medecins, Sans Frontieres, 2010; 11-64.

2. Maclvor D, Yazer M. Transfusion support in sickle cell anemia. Transfusion Medicine Update, Institute for Transfusion Medicine, 2007; 1-2.

3. Ozsoylu S. ABC of blood transfusion in patients with thalassemia major. Medical Journal of Islamic World Academy of Sciences, 2014; 22(4): 175-6.

4. UTDRS RSUD Dr Soetomo. Data permintaan darah transfusi dan komponennya. Surabaya, UTDRS RSUD Dr Soetomo, 2016.

5. Quienly E. Immunohematology principles and practice. $3^{\text {rd }}$ Ed., Washington DC, Lippincot Williams \& Wilkins, 2011; 107-18.

6. Norfolk D. Handbook of transfusion medicine. $5^{\text {th }}$ Ed., United Kingdom Blood Service,TSO, 2013; 5-11.
7. Lane D. Pre-transfusion testing. Clinical guide to transfusion. Canadian Blood Service, 2014; 1-3.

8. Delaney M, Wendel S, Bercovitz RS, Cid J, Cohn C, et al. Transfusion reactions: Prevention, diagnosis, and treatment. The Lancet, 2016; 388(10061): 2825-36.

9. Westhoff $C$. Red cell immunology and compatibility testing. Rossi's principle transfusion. $5^{\text {th }}$ Ed., New York, John Wiley \& Son, 2016; 193-205.

10. McCullough J. Transfusion medicine. $4^{\text {th }}$ Ed., John Wiley \& Son, 2017; 220-3.

11. EPHTI. The crossmatch (compatibility testing). In: Immunohaemotology. $1^{\text {st }}$ Ed., Misganaw Birhaneselassie, 2004; 79-84.

12. Malyska $H$, Weiland $D$. The gel test. Laboratory Medicine, 1994; 25(2): 81-5.

13. Cid J, Nogues N, Montero R, Hurtado M, Briega A, Parra R. Comparison of three microtube column agglutination systems for antibody screening: DG Gel, DiaMed-ID and Ortho BioVue. Transfusion Medicine, 2006; 16(2): 131-6.

14. Swarup D, Dhot P, Kotwal J, Verma A. Comparative study of blood cross matching using conventional tube and gel method. MJAFI, 2008; (64): 129-30.

15. Mehta N, Chakraborty I, Rane M, Ambre V. Verification of column agglutination technology with conventional tube technology for naturally occurring antibody titration. Global Journal of Transfusion Medicine, 2016; 1(2): 46.

16. Taylor J, Hyare J, Stelfox P, Williams M, Lees R, Maley M. Multi-centre evaluation of pre-transfusional routine tests using 8-column format gel cards (DG Gel $($ )). Transfusion Medicine, 2011; 21(2): 90-8.

17. Hustinx $H$, Munger $E$, Lejon Crottet $S$. Comparison of sensitivity and specificity of the Bio-Rad ID Cards LISS/Coombs with the Grifols Gel Coombs cards in a manual approach. Switzerland, Interregional Transfusion SRC Berne, 2016; 1.

18. Watkins J. Introduction to the science of statistics: From theory to implementation. Preelimina, 2005; 173-182,333-352.

19. Zammit V. A comparative study between antiglobulin crossmatch and type and screen procedures for compatibility testing. B.Sc. (Hons.) in Health Science, 2004; 52-94.

20. Diamed AG. Diamed-ID microtyping system ID Card "LISS/Coombs" indirect and direct antiglobulin test. Product Identification No 50531. Switzerland, Diamed AG, 2008; 13-22.

21. Diagnostic Grifols. DG Gel Coombs. Katalog No 031222. Spain, Diagnostic Grifols, 2010; 1-2.

22. McHugh $M$. Interrater reliability: The kappa statistic. Biochemia Medica, 2012; 22(3): 276-82.

23. Grey D, Connolly M, Erber W. Comparison of low ionic diluents for use with the Diamed antiglobulin test. Transfusion Medicine, 2002; 12: 63-9.

24. Majekodunmi S. A review on centrifugation in the pharmaceutical industry. American Journal of Biomedical Engineering, 2015; 5(2): 67-78. 